\title{
MS10-02 | ERrors in Electron SCATtering and Validation of Experimental MapS through Combination of MicroEd and CryoEM
}

Usón, Isabel (ICREA at IBMB-CSIC, Barcelona, ESP); Millán Nebot, Claudia Lucía (Institut de Biologia Molecular de Barcelona, Barcelona, ESP); Borges, Rafael (Crystallographic Methods, Institute of Molecular Biology of Barcelona (IBMB-CSIC), Barcelona, ESP)

Electron scattering is described in crystallography and EM in terms of spherical atoms, whereas the potential map determined depends both on the interactions with electrons and nuclei, and thus on the environment of each atom, not just on the scattering element. Advances in MicroED have opened exciting possibilities in the determination of structures from micro-crystals. We have adapted our fragment-based phasing methods to microED structures, incorporating maps as search fragments.

On borderline cases, we are exploring differences between the use of tabulated electron scattering factors to calculate the scattering from a helix, as we do for X-rays vs. cutting an helix out of a cryoEM map and thus resorting to the experimental scattering. This entails exploring the differences derived from using one map or another.

Within the ARCIMBOLDO framework, which combines fragment location with PHASER and density modification and autotracing with SHELXE, we are investigating both aspects: solution of microED data and examination of cryoEM map genesis, treatment and resolution through its performance against microED data. The potential of this approach lies in introducing an external experimental method as a validation probe for cryoEM. Are CryoEM maps currently too biased towards crystallography without accounting for a fundamental difference in scattering behaviour? 